\title{
Mathematical Analysis and Availability of the Pulping System in the Paper Industry
}

\author{
Zeenat Zaidi and Yogesh Kumar Goyal
}

\begin{abstract}
In this paper, we tried to find out the availability of the pulping system in the Paper Industry in the transient state. Markov method is applied and the systems of differential difference equations are formed. These equations are solved with the help of Matrix Method using Computer program. Long run availability is also calculated which is helpful for reliability engineers and managers. The parameter MTTF is also computed. The concept of Correlation and Regression are also applied to study the variation of availability with respect to time.
\end{abstract}

Index Terms-Availability, markov method, matrix method, correlation and regression.

\section{INTRODUCTION}

The availability function $A(\underline{t})$ is defined as the probability that the equipment is operating at time t. Availability depends upon both failure and repair rates. Availability is another measure of performance of maintained equipments. It integrates both reliability and maintainability parameters and depends on the number of failures that occur and on how quickly any faults are rectified. In this paper, we tried to find out the availability of pulping system in the Paper industry. Paper industry is a process industry as there is a sequence of processes. The paper production system consists of six subsystems namely feeding, pulping, washing, bleaching, screening and paper formation. Each subsystem can analysed to obtain overall capability of the paper industry. The mathematical analysis of each subsystem involves large number of differential difference equations. Laplace Transform method is easily applicable in solving equations for simple systems, but for complex systems, inversion of Laplace transforms is very difficult. In this paper we applied matrix method and a C-program is developed which is applicable to matrices of large order without evaluating the Eigen value. A pulping system of paper manufacturing plant, taking different sets of failure and repair rates is analysed for availability etc. Graphs are also drawn to see the behaviour of the system.

The concept of availability is widely discussed in literature and the main contributors are Barlow and Hunter [1960], Gaver [1963], Sandler [1963], Myers [1964], Barlow and Proschan [1965], Rau [1970]. Singh and Billington [1974, 1975] suggest methods for determining the frequency of failures of complex systems. Dhillon et al., [1] have frequently used the Markovian approach for the availability analysis, using exponential distribution for failure and repair

Manuscript received August 12, 2013; revised October 23, 2013.

Zeenat Zaidi is with Al- Qassim University, Kingdom of Saudi Arabia, K.S.A (e-mail: naqvizeenat@yahoo.co.in)

Yogesh kumar Goyal is with Aggarwal P. G. College Ballabgarh, Haryana, India. times. Kumar et al., [2]-[5] dealt with reliability, availability and operational behaviour analysis for different systems in the paper plant. Zhao [6] developed a generalized availability model for repairable component and series system including perfect and imperfect repair. Shooman [7] discussed the reliability computation for systems with dependent failures. Michelson [8] explained the use of reliability technology in process industry. Singh and Mahajan [9] examined the reliability and long run availability of a Utensils manufacturing Plant using Laplace Transforms. Castro and Cavalca [10] presented an availability optimization problem of an engineering system assembled in series configuration which has redundancy of units and teams of maintenance as optimization parameters. Tewari, Joshi and Rao [11] discussed about the mathematical modeling and behavioural analysis of a refining system using genetic algorithm. Gupta, Lal, Sharma and Singh [12] discussed the reliability, long term availability and MTBF of cement industry with the help of Runge-Kutta method. Singh and Goyal [13] discussed availability in Bread manufacturing plant. In these papers, authors used either Laplace transforms method or Runge-Kutta method to solve differential equations. Kiureghian and Ditlevson [14] analysed the availability, reliability and downtime of system with repairable components. Kumar, Singh and Sharma discussed the availability of an automobile system namely "scooty". Jussi K. Vaurio [15] discussed current research and application related to the modelling, optimization and application of maintenance procedures for ageing and deteriorating engineering and structural systems. It has been observed that calculation of availability in transient state is very difficult in complex systems. In fact, problem of calculating variation of availability with time has not satisfactorily been tackled till now. In this paper, we have developed the matrix method to solve differential equations in transient state. The Matrix Method provides an easy way to estimate the variation in system performance in terms of availability with respect to time and computer program is developed to calculate the time dependent availability. The time dependent availability of the pulping system in the Paper Industry is also shown with the help of graph. The concept of correlation and regression are also implied to study the relation of availability with time in the pulping system. Long run availability is also studied with the help of tables at different repair rates. By studying these tables we can find out which subsystem requires preventive maintenance to avoid any possible failures. The results of the present paper are useful for the paper manufacturers and reliability engineers. 


\section{THE SYSTEM OF PAPER INDUSTRY}

The paper production system consists of six subsystems:

\section{A. Feeding System}

It consists of a chain conveyor for carrying chips from store to digesters and blower with blowing units for pneumatic conveying of chips to the digesters.

\section{B. Pulping System}

It consists of digesters for cooking the pulp using $\mathrm{NaOH}$, $\mathrm{Na}_{2} \mathrm{~S}$ and steam, knotter (fiberizer) to remove the knots from the cooked pulp, decker for removing the black liquor from the cooked pulp and refiner to open the knots.

\section{Washing System}

It consists of screening unit for separating the unwanted foreign material from the pulp, cleaner for removing heavy material from the pulp and washer for removing chemicals through washing.

\section{Bleaching System}

It consists of filter for filtering the unbleached pulp and opener to open the fibers.

\section{E. Screening System}

It is composed of four subsystem, filter to remove black liquor, screen for removing the knots and other undesirable material, cleaner and mixer for cleaning the fibers and mixing of fresh water with the pulp and washer to wash the pulp for brightness.

\section{F. Paper Formation System}

It consists of fiber decomposition and water suction unit, pressing unit for ironing and smoothening the paper sheets and dryers for removing the moisture content from the paper sheets.

\section{PULPING SYSTEM}

The pulping is an important part of the mill. It consists of four subsystems.

\section{1) The digester $B_{1}$}

Here a mixture of wooden chips and $\mathrm{NaOH}+\mathrm{Na}_{2} \mathrm{~S}$ is heated by steam at. Failure of digester stops the cooking process and hence leads to system failure.

\section{2) The decker $B_{2}$}

It used to remove the black liquor from the pulp. Failure of this causes complete failure of the process of block liquor removal.

\section{3) The knotter $B_{3}$}

It consists of one main unit and one stand by. Complete failure occurs on failure of both the units.

\section{4) The opener $B_{4}$}

It consists of one main unit and one standby. Complete failure of this subsystem occurs when both the unit fail.

\section{ASSUMPTIONS \& NOTATIONS}

- Repairs and failures are independent of each other.
- Simultaneous failures do not occur at a time.

- Repaired Systems are like new systems.

- The subsystems $B_{3}$ and $B_{4}$ fails only through reduced states.

- Repair is carried out only when the subsystems are in reduced or failed state.

$B_{1}, B_{2}, B_{3}, B_{4}$ : Denote are good state of the systems.

$\overline{B_{3}} \& \overline{B_{4}}$ : Indicate that the subsystems $B_{3} \& B_{4}$ are working in reduced capacity.

$B_{1}, B_{2}, B_{3}, B_{4}$ : Represents the failure state of the subsystems $B_{1}, B_{2}, \overline{B_{3}}, \overline{B_{4}}$.

$\lambda_{i}(1 \leq i \leq 6)$ : represents failure rate of the subsystems $B_{1}, B_{2}, B_{3} \overline{B_{3}}, B_{4}, \overline{B_{4}}$.

$\mu_{i}(1 \leq i \leq 6)$ : represents respective repair rate of $B_{1}, B_{2}, B_{3} \overline{B_{3}}, B_{4}, \overline{B_{4}}$.

$P_{i}(t)=P(i, t):$ denotes probability that the system is in i-th state at time $t(0 \leq i \leq 15)$.

Following the above notations and assumptions, the transition diagram is as shown in Fig. 1.
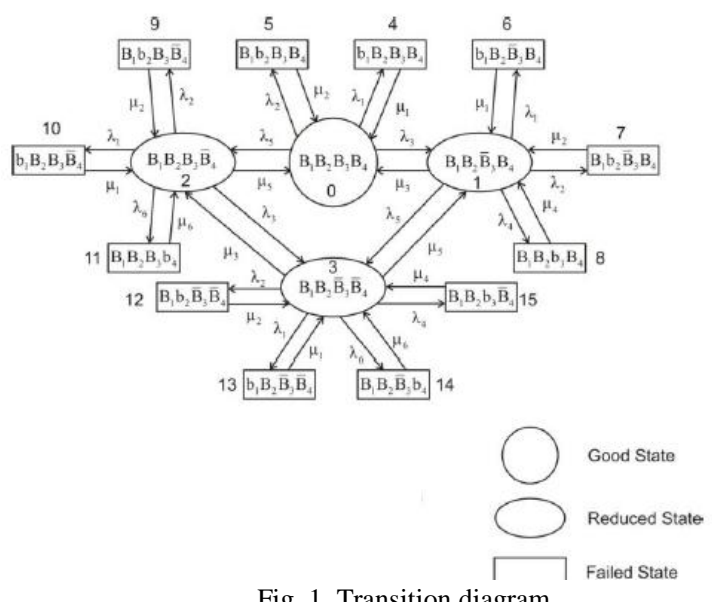

\section{MATHEMATICAL FORMULATION AND SOLUTION}

The probability consideration gives the following differential equations, associated with the

$$
\begin{gathered}
P_{0}^{\prime}(t)+\left(\lambda_{1}+\lambda_{2}+\lambda_{3}+\lambda_{5}\right) P_{0}(t) \\
=\mu_{1} P_{4}(t)+\mu_{2} P_{5}(t)+\mu_{3} P_{1}(t)+\mu_{5} P_{2}(t) \\
P_{1}^{\prime}(t)+\left(\lambda_{1}+\lambda_{2}+\lambda_{4}+\lambda_{5}+\mu_{3}\right) P_{1}(t) \\
=\mu_{1} \mathrm{P}_{6}(\mathrm{t})+\mu_{2} P_{7}(t)+\mu_{4} P_{8}(t)+\mu_{5} P_{3}(t)+\lambda_{3} P_{0}(t) \\
P_{2}^{\prime}(t)+\left(\lambda_{1}+\lambda_{2}+\lambda_{3}+\lambda_{6}+\mu_{5}\right) P_{2}(t) \\
=\mu_{1} \mathrm{P}_{10}(\mathrm{t})+\mu_{2} P_{9}(t)+\mu_{3} P_{3}(t)+\mu_{6} P_{11}(t)+\lambda_{5} P_{0}(t) \\
P_{3}^{\prime}(t)+\left(\lambda_{1}+\lambda_{2}+\lambda_{4}+\lambda_{6}+\mu_{3}+\mu_{5}\right) \mathrm{P}_{3}(\mathrm{t})=\mu_{1} P_{13}(t) \\
+\mu_{2} P_{11}(t)+\mu_{4} P_{15}(t)+\mu_{6} P_{14}(t)+\lambda_{3} P_{2}(t)+\lambda_{5} P_{1}(t) \\
P_{4}^{\prime}(t)+\mu_{1} P_{4}(t)=\lambda_{1} P_{0}(t) \\
P_{5}^{\prime}(t)+\mu_{2} P_{5}(t)=\lambda_{2} P_{0}(t)
\end{gathered}
$$




$$
\begin{array}{r}
P_{6}^{\prime}(t)+\mu_{1} P_{6}(t)=\lambda_{1} P_{1}(t) \\
P_{7}^{\prime}(t)+\mu_{2} P_{7}(t)=\lambda_{2} P_{1}(t) \\
P_{8}^{\prime}(t)+\mu_{4} P_{8}(t)=\lambda_{4} P_{1}(t) \\
P_{9}^{\prime}(t)+\mu_{2} P_{9}(t)=\lambda_{2} P_{2}(t) \\
P_{10}^{\prime}(t)+\mu_{1} P_{10}(t)=\lambda_{1} P_{2}(t) \\
P_{11}^{\prime}(t)+\mu_{6} P_{11}(t)=\lambda_{6} P_{2}(t) \\
P_{12}^{\prime}(t)+\mu_{2} P_{12}(t)=\lambda_{2} P_{3}(t) \\
P_{13}^{\prime}(t)+\mu_{1} P_{13}(t)=\lambda_{1} P_{3}(t) \\
P_{14}^{\prime}(t)+\mu_{6} P_{14}(t)=\lambda_{6} P_{3}(t) \\
P_{15}^{\prime}(t)+\mu_{4} P_{15}(t)=\lambda_{4} P_{3}(t)
\end{array}
$$

where

$$
\begin{gathered}
\mathrm{A}_{1}=+\left(\lambda_{1}+\lambda_{2}+\lambda_{3}+\lambda_{5}\right) \\
\mathrm{A}_{2}=+\left(\lambda_{1}+\lambda_{2}+\lambda_{4}+\lambda_{5}+\mu_{3}\right) \\
\mathrm{A}_{3}=+\left(\lambda_{1}+\lambda_{2}+\lambda_{3}+\lambda_{6}+\mu_{5}\right) \\
\mathrm{A}_{4}=+\left(\lambda_{1}+\lambda_{2}+\lambda_{4}+\lambda_{6}+\mu_{3}+\mu_{5}\right)
\end{gathered}
$$

With initial conditions $P_{0}(0)=1$, otherwise zero.

Time Dependent Case: The availability of the system is obtained by solving the matrix differential difference equations

$(\theta \mathrm{I}-\mathrm{A}) \bar{P}_{i}(t)=\mathrm{O}$, where $\theta \equiv d / d t, \mathrm{O}$ is the null matrix, matrix $\mathrm{A}$ is the matrix of the coefficients of the probability states $p_{i}(t)$ and $I$ or $I_{n}$ is the identity matrix of order $n$.

$$
\begin{aligned}
& \mathrm{A}= \\
& {\left[\begin{array}{cccccccccccccccc}
-\mathrm{A}_{1} & \mu_{3} & \mu_{5} & 0 & \mu_{1} & \mu_{2} & 0 & 0 & 0 & 0 & 0 & 0 & 0 & 0 & 0 & 0 \\
\lambda_{3} & -\mathrm{A}_{2} & 0 & \mu_{5} & 0 & 0 & \mu_{1} & \mu_{2} & \mu_{4} & 0 & 0 & 0 & 0 & 0 & 0 & 0 \\
\lambda_{5} & 0 & -\mathrm{A}_{3} & \mu_{3} & 0 & 0 & 0 & 0 & 0 & \mu_{2} & \mu_{1} & \mu_{6} & 0 & 0 & 0 & 0 \\
0 & \lambda_{5} & \mu_{3} & -\mathrm{A}_{4} & 0 & 0 & 0 & 0 & 0 & 0 & 0 & 0 & \mu_{2} & \mu_{1} & \mu_{6} & \mu_{4} \\
\lambda_{1} & 0 & 0 & 0 & -\mu_{1} & 0 & 0 & 0 & 0 & 0 & 0 & 0 & 0 & 0 & 0 & 0 \\
\lambda_{2} & 0 & 0 & 0 & 0 & -\mu_{2} & 0 & 0 & 0 & 0 & 0 & 0 & 0 & 0 & 0 & 0 \\
0 & \lambda_{1} & 0 & 0 & 0 & 0 & -\mu_{1} & 0 & 0 & 0 & 0 & 0 & 0 & 0 & 0 & 0 \\
0 & \lambda_{2} & 0 & 0 & 0 & 0 & 0 & -\mu_{2} & 0 & 0 & 0 & 0 & 0 & 0 & 0 & 0 \\
0 & \lambda_{4} & 0 & 0 & 0 & 0 & 0 & 0 & -\mu_{4} & 0 & 0 & 0 & 0 & 0 & 0 & 0 \\
0 & 0 & \lambda_{2} & 0 & 0 & 0 & 0 & 0 & 0 & -\mu_{2} & 0 & 0 & 0 & 0 & 0 & 0 \\
0 & 0 & \lambda_{1} & 0 & 0 & 0 & 0 & 0 & 0 & 0 & -\mu_{1} & 0 & 0 & 0 & 0 & 0 \\
0 & 0 & \lambda_{6} & 0 & 0 & 0 & 0 & 0 & 0 & 0 & 0 & -\mu_{6} & 0 & 0 & 0 & 0 \\
0 & 0 & 0 & \lambda_{2} & 0 & 0 & 0 & 0 & 0 & 0 & 0 & 0 & -\mu_{2} & 0 & 0 & 0 \\
0 & 0 & 0 & \lambda_{1} & 0 & 0 & 0 & 0 & 0 & 0 & 0 & 0 & 0 & -\mu_{1} & 0 & 0 \\
0 & 0 & 0 & \lambda_{6} & 0 & 0 & 0 & 0 & 0 & 0 & 0 & 0 & 0 & 0 & -\mu_{6} & 0 \\
0 & 0 & 0 & \lambda_{4} & 0 & 0 & 0 & 0 & 0 & 0 & 0 & 0 & 0 & 0 & 0 & -\mu_{2}
\end{array}\right]}
\end{aligned}
$$

\section{AVAILABILITY ANALYSIS}

The availability of the system is obtained by solving the matrix differential difference equations $(\theta I-\mathrm{A}) \bar{P}_{i}(t)=\mathrm{O}$, where $\theta \equiv d / d t$, $\mathrm{O}$ is the null matrix, matrix $\mathrm{A}$ is the matrix of the coefficients of the probability states $p_{i}(t)$ and $I$ or $I_{n}$ is the identity matrix of order $n$.

The equations reduce to $C^{-1}(\theta I-\mathrm{D}) \bar{P}_{i}(t)=\mathrm{O}$, where $\mathrm{C}$ is the matrix such that $\mathrm{C}^{-1} \mathrm{AC}=\mathrm{D}$, and $\mathrm{D}=\left(d_{1}, d_{2}, \ldots, d_{n}\right)$ is the matrix of Eigen values of the matrix A (Table II, Table III) .

The availability of the system is sum of the availabilities of working subsystems.

$$
\begin{gathered}
\operatorname{Av}(t)=P_{0}(t)+P_{1}(t)+P_{2}(t)+P_{3}(t) \\
=1+\left(a_{11}+a_{21}+a_{31}+a_{41}\right) t \\
+\left(b_{11}+b_{21}+b_{31}+b_{41}\right) t^{2} / 2 !+\ldots
\end{gathered}
$$

Availability of the Pulping system at time $t$ is,

$$
\begin{aligned}
& \mathrm{A} v(t)=P(0, t)+\mathrm{P}(1, t)+\mathrm{P}(2, t)+\mathrm{P}(3, t) \\
= & 1-0.030000 t+0.000875 t^{2}-0.0000023 t^{3}+\ldots . .
\end{aligned}
$$

The graph of the availability is as shown in Fig. 2.

Mean Time to Failure (MTTF): The MTTF of the system for these failure and repair rates is obtained by integrating the availability function of the system.

MTTF $=t-0.030000 \frac{t^{2}}{2}+0.000875 \frac{t^{3}}{3}-0.0000023 \frac{t^{4}}{4}+\ldots$

The table of availability is drawn at different time interval by taking the failure and repair rates as

$$
\begin{aligned}
& \lambda_{1}=0.001, \lambda_{2}=0.002, \lambda_{3}=0.002, \\
& \lambda_{4}=0.0015, \lambda_{5}=0.0015, \lambda_{6}=0.002 \\
& \mu_{1}=0.002, \mu_{2}=0.015, \mu_{3}=0.002, \\
& \mu_{4}=0.02, \mu_{5}=0.012, \mu_{6}=0.002
\end{aligned}
$$

The failure and repair rates are taken monthly.

TABLE I: THE VARIATION OF AVAILABILITY WITH RESPECT TO TIME

\begin{tabular}{llllll}
\hline \hline Time & $\mathbf{1 0}$ & $\mathbf{2 0}$ & $\mathbf{3 0}$ & $\mathbf{4 0}$ & $\mathbf{5 0}$ \\
Availability & 0.971731 & 0.946824 & 0.925080 & 0.906272 & 0.890161 \\
\hline Time & $\mathbf{6 0}$ & $\mathbf{7 0}$ & $\mathbf{8 0}$ & $\mathbf{9 0}$ & $\mathbf{1 0 0}$ \\
Availability & 0.876516 & 0.865124 & 0.855804 & 0.848435 & 0.843010 \\
\hline \hline
\end{tabular}

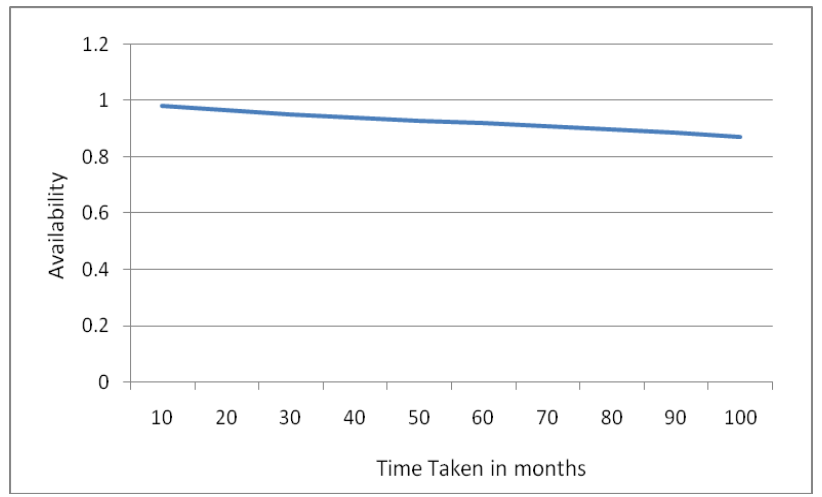

Fig. 2. The variation of availability with respect to time.

The Program used:

$$
\begin{gathered}
\lambda_{1}=.001 \quad \mu_{2}=.002 \quad \mathrm{~A}_{1}=.0065 \quad \mathrm{~A}_{3}=.019 \\
\lambda_{2}=\lambda_{3}=\lambda_{6}=.002 \quad \mu_{1}=\mu_{3}=\mu_{6}=.002 \quad \mathrm{~A}_{2}=.0080 \\
\mathrm{~A}_{4}=.0205 \\
\lambda_{4}=\lambda_{5}=.0015 \quad \mu_{4}=.02 \quad \mu_{5}=.012
\end{gathered}
$$

\#include $<$ stdio.h $>$

\#include $<$ conio.h $>$

void main()

\{

float $a_{1}[16][16], \mathrm{b}[16][16], \mathrm{c}[16][16], d[16][16], e[16][16], f$

[16][16];

float $g[16][16], \mathrm{h}[16][16], o[16][16], p[16][16], q[16][16], r$ 
[16][16];

float $x_{1}, x_{2}, x_{3}, x_{4}, x_{5}, x_{6}, x_{7}, x_{8}, x_{9}, x_{10}, x_{11}, x_{12}, x$;

int $i, j, k, 1, n=16, m=16, t=10$;

float $a[16][16]=$

$\{-.0065, .002, .012,0, .002, .015,0,0,0,0,0,0,0,0,0,0$, $.002,-.0080,0, .012,0,0, .02, .002, .015,0,0,0,0,0,0,0$,

$.0015,0,-.019, .002,0,0,0,0,0, .002, .002, .015,0,0,0,0$,

$0, .0015, .002,-.0205,0,0,0,0,0,0,0,0, .002, .015, .02, .002$,

$.001,0,0,0,-.002,0,0,0,0,0,0,0,0,0,0,0$,

$.002,0,0,0,0,-.015,0,0,0,0,0,0,0,0,0,0$,

$0, .0015,0,0,0,0,-.02,0,0,0,0,0,0,0,0,0$,

$0, .001,0,0,0,0,0,-.002,0,0,0,0,0,0,0,0$,

$0, .002,0,0,0,0,0,0,-.015,0,0,0,0,0,0,0$,

$0,0, .002,0,0,0,0,0,0,-.002,0,0,0,0,0,0$,

$0,0, .001,0,0,0,0,0,0,0,-.002,0,0,0,0,0$,

$0,0, .020,0,0,0,0,0,0,0,0,-.015,0,0,0,0$,

$0,0,0, .001,0,0,0,0,0,0,0,0,-.002,0,0,0$,

$0,0,0, .002,0,0,0,0,0,0,0,0,0,-.015,0,0$,

$0,0,0, .0015,0,0,0,0,0,0,0,0,0,0,-.02,0$,

$0,0,0, .002,0,0,0,0,0,0,0,0,0,0,0,-.002\}$;

$\operatorname{clrscr}()$;

for $(i=0 ; i<m ; i++)$

\{

For $(j=0 ; j<n ; j++)$

\{

$b[i][j]=a[i][j]$;

\}

\}

for $(i=0 ; i<m ; i++)$

\{

for $(j=0 ; j<1 ; j++)$

\{

$c[i][j]=0$;

for $(1=0 ; 1<m ; 1++)$

$c[i][j]=(((a[i][1] \times \mathrm{b}[1][j]) \times t) / 2)+c[i][j] ;$

\}

\}

For $(i=0 ; i<m ; i++)$

\{

for $(j=0 ; j<1 ; j++)$

\{

$d[i][j]=0$

for $(1=0 ; 1<m ; 1++)$

$d[i][j]=(((a[i][1] \times c[1][j]) \times t) / 3)+d[i][j] ;$

\}

\}

for $(i=0 ; i<m ; i++)$

\{

for $(j=0 ; j<1 ; j++)$

\{

$e[i][j]=0$;

for $(1=0 ; 1<m ; 1++)$

$e[i][j]=(((a[i][1] \times \mathrm{d}[1][j]) \times t) / 4)+e[i][j] ;$

\}

\}

for $(i=0 ; i<m ; i++)$

\{

For $(j=0 ; j<1 ; j++)$

\{ $f[i][j]=0$;

for $(1=0 ; 1<m ; 1++)$

$f[i][j]=(((a[i][1] \times e[1][j]) \times t) / 5)+f[i][j]$;

\}

\}

for $(i=0 ; i<m ; i++)$

\{

For $(j=0 ; j<1 ; j++)$

\{

$\mathrm{g}[i][j]=0$;

for $(1=0 ; 1<m ; 1++)$

$g[i][j]=(((a[i][1] \times f[1][j]) \times t) / 6)+g[i][j] ;$

\}

\}

for $(i=0 ; i<m ; i++)$

\{

for $(j=0 ; j<1 ; j++)$

\{

$h[i][j]=0$;

for $(1=0 ; 1<m ; 1++)$

$h[i][j]=(((a[i][1] \times g[1][j]) \times t) / 7)+h[i][j] ;$

\}

\}

for $(i=0 ; i<m ; i++)$

\{

for $(j=0 ; j<1 ; j++)$

\{

$\mathrm{o}[i][j]=0$;

for $(1=0 ; 1<m ; 1++)$

$\mathrm{o}[i][j]=(((a[i][1] \times h[1][j]) \times t) / 8)+\mathrm{o}[i][j]$;

\}

printf("\n");

\}

for $(i=0 ; i<m ; i++)$

\{

for $(j=0 ; j<1 ; j++)$

\{

$p[i][j]=0$;

for $(1=0 ; 1<m ; 1++)$

$p[i][j]=(((a[i][1] \times \mathrm{o}[1][j]) \times t) / 9)+p[i][\mathrm{j}]$;

\}

\}

for $(i=0 ; i<m ; i++)$

\{

for $(j=0 ; j<1 ; j++)$

\{

$q[i][j]=0$;

for $(1=0 ; 1<m ; 1++)$

$q[i][j]=(((a[i][1] \times p[1][j]) \times t) / 10)+q[i][j] ;$

\}

\}for $(i=0 ; i<m ; i++)$

\{

For $(j=0 ; j<1 ; j++)$

\{

$r[i][j]=0$;

for $(1=0 ; 1<m ; 1++)$

$r[i][j]=(((a[i][1] \times q[1][j]) \times t) / 11)+r[i][j] ;$

\}

\} 
$x_{1}=((a[0][0]+a[1][0]+a[2][0]+a[3][0]) \times t) ;$

printf("\} \backslash n \mathrm { A } 1 1 = \% \mathrm { f } " , x _ { 1 } ) \text { ; }

$x_{2}=((c[0][0]+c[1][0]+c[2][0]+c[3][0]) \times t)$;

printf(" $\left.\ln \backslash n \mathrm{C} 11=\% \mathrm{f} ", x_{2}\right)$;

$x_{3}=((d[0][0]+d[1][0]+d[2][0]+d[3][0]) \times \mathrm{t}) ;$

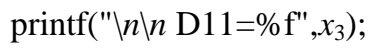

$x_{4}=((e[0][0]+e[1][0]+e[2][0]+e[3][0]) \times t)$;

printf(" $\left.\backslash n \backslash n \mathrm{E} 11=\% \mathrm{f} ", x_{4}\right)$;

$x_{5}=((f[0][0]+f[1][0]+f[2][0]+f[3][0]) \times t) ;$

printf("\n\n F11=\%f", $\left.x_{5}\right)$;

$x_{6}=((g[0][0]+g[1][0]+g[2][0]+g[3][0]) \times t)$;

printf("\n\n G11=\%f", $\left.x_{6}\right)$;

$x_{7}=((h[0][0]+h[1][0]+h[2][0]+h[3][0]) \times t)$;

printf(" $\left.\backslash n \backslash n \mathrm{H} 11=\% \mathrm{f} ", x_{7}\right)$;

$x_{8}=((o[0][0]+o[1][0]+o[2][0]+o[3][0]) \times t)$;

printf(" $\left.\ln n \mathrm{O} 11=\% \mathrm{f} ", x_{8}\right)$;

$x_{9}=((p[0][0]+p[1][0]+p[2][0]+p[3][0]) \times t)$;

printf("\n\n P11=\%f", $\left.x_{9}\right)$;

$x_{10}=((q[0][0]+r[1][0]+q[2][0]+q[3][0]) \times t)$;

printf("In $\left.\backslash n \mathrm{Q} 11=\% \mathrm{f} ", x_{10}\right)$;

$x_{11}=((r[0][0]+r[1][0]+r[2][0]+r[3][0]) \times t)$;

$\operatorname{printf}\left(" \backslash n \backslash n \mathrm{R} 11=\% \mathrm{f} ", x_{11}\right)$;

printf(" $\backslash n \backslash n \backslash n f(t)=1+\% f+\% f+\% f+\% f+\% f+\% f+\% f$

$\left.+\% f+\% f+\% f+\% f \% f f \% f \backslash n ", x_{1}, x_{2}, x_{3}, x_{4}, x_{5}, x_{6}, x_{7}, x_{8}, x_{9}, x_{10}, x_{11}\right)$;

$x=1+x_{1}+x_{2}+x_{3}+x_{4}+x_{5}+x_{6}+x_{7}+x_{8}+x_{9}+x_{10}+x_{11} ;$

printf("\n\n $x=\% f ", \underline{x})$;

getch();

\}

TABLE II: CORRELATION BETWEEN TIME AND AVAILABILITY

\begin{tabular}{|c|c|c|c|c|c|c|}
\hline$x$ & $y$ & $\begin{array}{c}X= \\
\bar{x} \\
-\bar{x}\end{array}$ & $\begin{array}{r}Y= \\
y-\bar{y}\end{array}$ & $X^{2}$ & $Y^{2}$ & $X Y$ \\
\hline 10 & 0.971731 & -45 & 0.071731 & 2025 & 0.00514533 & -3.227895 \\
\hline 20 & 0.946824 & -35 & 0.046824 & 1225 & 0.00219248 & -1.63884 \\
\hline 30 & 0.925080 & -25 & 0.025080 & 625 & 0.00062900 & -0.62700 \\
\hline 40 & 0.906272 & -15 & 0.006272 & 225 & 0.00003933 & -0.15680 \\
\hline 50 & 0.890161 & -5 & -0.009839 & 25 & 0.00009680 & 0.04919 \\
\hline 60 & 0.876516 & 5 & -0.023484 & 25 & 0.00055149 & -0.11742 \\
\hline 70 & 0.865124 & 15 & -0.034876 & 225 & 0.00121633 & -0.52314 \\
\hline 80 & 0.855804 & 25 & -0.044196 & 625 & 0.00195328 & -1.10490 \\
\hline 90 & 0.848435 & 35 & -0.051565 & 1225 & 0.00265840 & -1.80460 \\
\hline 100 & 0.843010 & 45 & -0.056990 & 2025 & 0.00324786 & -2.56455 \\
\hline$\Sigma x=$ & $\Sigma y=$ & & & $\Sigma X^{2}=$ & $\Sigma Y^{2}=$ & $\Sigma x y=$ \\
\hline 550 & 8.93 & & & 8300 & 0.1777284 & -11.71581 \\
\hline
\end{tabular}

$\bar{x}=55, \bar{y}=0.893=0.9$ (approx.)

Coefficient of correlation $r=\frac{\Sigma x y}{\sqrt{\Sigma x^{2} \cdot \Sigma y^{2}}}=-0.3050$

Result: Time and Availability are negatively correlated to each other.

Calculating Regression coefficients $b_{y x}$ and $b_{x y}-$

Regression coefficient $(y$ on $x$ )

$$
b_{y x}=\frac{n \sum x y-\Sigma x \Sigma y}{n \sum x^{2}-(\Sigma x)^{2}}=0.00142
$$

Regression line of $y$ on $x$ :

$$
(y-\bar{y})=b_{y x}(x-\bar{x})
$$

Putting the values, we get $y=-0.00142 x+$ 0.9711Regression coefficient $(x$ on $y)$

$$
b_{x y}=\frac{n \Sigma \mathrm{xy}-\Sigma \mathrm{x} \Sigma y}{n \Sigma y^{2}-(\Sigma y)^{2}}=-764.68
$$

Regression line of $\mathrm{x}$ on $\mathrm{y}$ :

$$
(x-\bar{x})=b_{x y}(y-\bar{y})
$$

Putting the values, we get $x=-764.68 y+737.85$

The two lines of regression give the relationship between Time and Availability. Regression analysis is widely used for prediction and is also used to understand which among the independent variables are related to the dependent variable, and to explore the forms of these relationships (Table IV, Table V).

TABLE III: REgRESSION ANALYSIS BETWEEN TIME AND AVAILABILITY

\begin{tabular}{lllll}
\hline \hline$x$ & $y$ & $x y$ & $x^{2}$ & $y^{2}$ \\
\hline 10 & 0.971731 & 9.71731 & 100 & 0.94426 \\
20 & 0.946824 & 18.93648 & 400 & 0.89647 \\
30 & 0.925080 & 27.75240 & 900 & 0.85577 \\
40 & 0.906272 & 36.25088 & 1600 & 0.82132 \\
50 & 0.890161 & 44.50805 & 2500 & 0.79238 \\
60 & 0.876516 & 52.59096 & 3600 & 0.76828 \\
70 & 0.865124 & 60.55868 & 4900 & 0.74843 \\
80 & 0.855804 & 68.46432 & 6400 & 0.73240 \\
90 & 0.848435 & 76.35915 & 8100 & 0.71984 \\
100 & 0.843010 & 84.30100 & 10000 & 0.71066 \\
\hline$\Sigma x=$ & $\Sigma y=8.93$ & $\sum x y=$ & $\Sigma x^{2}=$ & $\Sigma y^{2}=$ \\
550 & & 479.435 & 38500 & 7.98981 \\
\hline \hline $\bar{x}=55, \bar{y}=0.893=0.9$ (approx. $)$ & &
\end{tabular}

\section{LONG RUN AVAILABILITY}

The management is always interested in long run availability of the system. Long run availability may be calculated by considering the fact that $\frac{d}{d t} \rightarrow 0$ as $t \rightarrow \infty$. The differential difference equations reduce to:

$$
\begin{gathered}
+\mathrm{A}_{1} P_{0}=\mu_{1} P_{4}+\mu_{2} P_{5}+\mu_{3} P_{1}+\mu_{5} P_{2} \\
+\mathrm{A}_{2} P_{1}=\mu_{1} P_{6}+\mu_{2} P_{7}+\mu_{4} P_{8}+\mu_{5} P_{3}+\lambda_{3} P_{0} \\
+\mathrm{A}_{3} P_{2}=\mu_{1} P_{10}+\mu_{2} P_{9}+\mu_{3} P_{3}+\mu_{6} P_{11}+\lambda_{5} P_{0} \\
+\mathrm{A}_{4} P_{3}=\mu_{1} P_{13}+\mu_{2} P_{12}+\mu_{4} P_{15}+\mu_{6} P_{14}+\lambda_{3} P_{2}+\lambda_{3} P_{1} \\
\mu_{1} P_{4}=\lambda_{1} P_{0} \\
\mu_{2} P_{5}=\lambda_{2} P_{0} \\
\mu_{1} P_{6}=\lambda_{1} P_{1} \\
\mu_{2} P_{7}=\lambda_{2} P_{1} \\
\mu_{4} P_{8}=\lambda_{4} P_{1} \\
\mu_{2} P_{9}=\lambda_{2} P_{2}
\end{gathered}
$$




$$
\begin{aligned}
& \mu_{1} P_{10}=\lambda_{1} P_{2} \\
& \mu_{6} P_{11}=\lambda_{6} P_{2} \\
& \mu_{2} P_{12}=\lambda_{2} P_{3} \\
& \mu_{1} P_{13}=\lambda_{1} P_{3} \\
& \mu_{6} P_{14}=\lambda_{6} P_{3} \\
& \mu_{4} P_{15}=\lambda_{4} P_{3}
\end{aligned}
$$

$$
\begin{aligned}
& \left(\lambda_{5}+\mu_{3}\right) P_{1}=\mu_{5} P_{3}+\lambda_{3} P_{0} \\
& \left(\lambda_{3}+\mu_{5}\right) P_{2}=\mu_{3} P_{3}+\lambda_{5} P_{0} \\
& \left(\mu_{3}+\mu_{5}\right) P_{3}=\lambda_{3} P_{2}+\lambda_{5} P_{1}
\end{aligned}
$$

\begin{tabular}{|c|c|c|c|c|c|c|c|c|c|}
\hline $\begin{array}{c}\mu_{1} \rightarrow \\
\mu_{2} \downarrow\end{array}$ & 0.1 & 0.15 & 0.2 & 0.25 & 0.3 & 0.35 & 0.4 & 0.45 & 0.5 \\
\hline 0.10 & 0.832171 & 0.855914 & 0.868300 & 0.875906 & 0.881050 & 0.884762 & 0.887567 & 0.889761 & 0.891523 \\
\hline 0.15 & 0.855914 & 0.881050 & 0.894181 & 0.902248 & 0.907708 & 0.911649 & 0.914627 & 0.916956 & 0.918829 \\
\hline 0.20 & 0.868300 & 0.894181 & 0.907708 & 0.916023 & 0.921651 & 0.925714 & 0.928785 & 0.931187 & 0.933118 \\
\hline 0.25 & 0.875906 & 0.902248 & 0.916023 & 0.924492 & 0.930225 & 0.934364 & 0.937492 & 0.939940 & 0.941907 \\
\hline 0.30 & 0.881050 & 0.907708 & 0.921651 & 0.930225 & 0.936030 & 0.940220 & 0.943388 & 0.945867 & 0.947859 \\
\hline 0.35 & 0.884762 & 0.911649 & 0.925714 & 0.934364 & 0.940220 & 0.944449 & 0.947645 & 0.950146 & 0.952157 \\
\hline 0.40 & 0.887567 & 0.914627 & 0.928785 & 0.937492 & 0.943388 & 0.947645 & 0.950863 & 0.953382 & 0.955406 \\
\hline 0.45 & 0.889761 & 0.916956 & 0.931187 & 0.939940 & 0.945867 & 0.950146 & 0.953382 & 0.955913 & 0.957948 \\
\hline 0.50 & 0.891523 & 0.918829 & 0.933118 & 0.941907 & 0.947859 & 0.952157 & 0.955406 & 0.957948 & 0.959992 \\
\hline
\end{tabular}

Solving these equation recursively, we get

First four equation using rest of equation becomes.

$$
P_{1}=\frac{\lambda_{3}}{\mu_{3}} P_{0}, P_{2}=\frac{\lambda_{5}}{\mu_{5}} P_{0}, P_{3}=\frac{\lambda_{5} \lambda_{3}}{\mu_{3} \mu_{5}} P_{0}
$$

$$
\left(\lambda_{3}+\lambda_{5}\right) P_{0}=\mu_{3} P_{1}+\mu_{5} P_{2}
$$

TABLE IV: REPAIR RATE OF KNOTTER INSURES MORE AVAILABILITY IN COMPARISON TO REPAIR RATE OF OPENER

\begin{tabular}{llllllllll}
\hline \hline $\begin{array}{l}\mu_{3} \rightarrow \\
\mu_{5} \downarrow\end{array}$ & $\mathbf{0 . 1}$ & $\mathbf{0 . 1 5}$ & $\mathbf{0 . 2}$ & $\mathbf{0 . 2 5}$ & $\mathbf{0 . 3}$ & $\mathbf{0 . 3 5}$ & $\mathbf{0 . 4}$ & $\mathbf{0 . 4 5}$ & $\mathbf{0 . 5}$ \\
\hline $\mathbf{0 . 1}$ & 0.832171 & 0.832445 & 0.832592 & 0.832683 & 0.832745 & 0.832790 & 0.832825 & 0.832852 & 0.832874 \\
$\mathbf{0 . 1 5}$ & 0.832256 & 0.832530 & 0.832677 & 0.832768 & 0.832830 & 0.832876 & 0.832910 & 0.832937 & 0.832959 \\
$\mathbf{0 . 2}$ & 0.832299 & 0.832573 & 0.839720 & 0.832811 & 0.832873 & 0.832919 & 0.839953 & 0.832980 & 0.833002 \\
$\mathbf{0 . 2 5}$ & 0.832325 & 0.832599 & 0.832745 & 0.832837 & 0.832899 & 0.832944 & 0.832979 & 0.833006 & 0.833027 \\
$\mathbf{0 . 3}$ & 0.832342 & 0.832616 & 0.832763 & 0.832854 & 0.832916 & 0.832962 & 0.832996 & 0.833023 & 0.833045 \\
$\mathbf{0 . 3 5}$ & 0.832355 & 0.832628 & 0.832775 & 0.832866 & 0.832929 & 0.832974 & 0.833008 & 0.833035 & 0.833057 \\
$\mathbf{0 . 4}$ & 0.832364 & 0.832638 & 0.832784 & 0.832876 & 0.832938 & 0.832983 & 0.833018 & 0.833045 & 0.833066 \\
$\mathbf{0 . 4 5}$ & 0.832371 & 0.832645 & 0.832792 & 0.832883 & 0.832945 & 0.832990 & 0.833025 & 0.833052 & 0.833073 \\
$\mathbf{0 . 5}$ & 0.832377 & 0.832651 & 0.832797 & 0.832888 & 0.832951 & 0.832996 & 0.833030 & 0.833057 & 0.833079 \\
\hline \hline
\end{tabular}

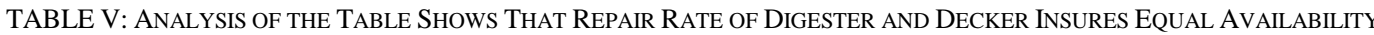

Availability $=P_{0}+P_{1}+P_{2}+P_{3}$

$$
\begin{aligned}
& =\left(1+\frac{\lambda_{3}}{\mu_{3}} P_{0}+\frac{\lambda_{5}}{\mu_{5}} P_{0}+\frac{\lambda_{5} \lambda_{3}}{\mu_{3} \mu_{5}} P_{0}\right) \\
& =\left(1+\frac{\lambda_{3}}{\mu_{3}}+\frac{\lambda_{5}}{\mu_{5}}+\frac{\lambda_{5} \lambda_{3}}{\mu_{5} \mu_{3}}\right) P_{0}
\end{aligned}
$$

Using normalizing condition $\sum_{i=0}^{15} P_{i}=1$

$$
\begin{gathered}
P_{0}+P_{1}+P_{2}+P_{3}+P_{4}+P_{5}+P_{6}+P_{7}+P_{8} \\
+P_{9}+P_{10}+P_{11}+P_{12}+P_{13}+P_{14}+P_{15}=1 \\
P_{0}=(1+a+b+c+d+e+f+g+h+i+j+k+1+m+n+\mathrm{o})^{-1} \\
=\left(1+\frac{\lambda_{3}}{\mu_{3}}+\frac{\lambda_{5}}{\mu_{5}}+\frac{\lambda_{3} \lambda_{5}}{\mu_{3} \mu_{5}}+\frac{\lambda_{1}}{\mu_{1}}+\frac{\lambda_{2}}{\mu_{2}}+\frac{\lambda_{1} \lambda_{3}}{\mu_{1} \mu_{3}}+\frac{\lambda_{2} \lambda_{3}}{\mu_{2} \mu_{3}}\right. \\
+\frac{\lambda_{3} \lambda_{4}}{\mu_{3} \mu_{4}}+\frac{\lambda_{2} \lambda_{5}}{\mu_{2} \mu_{5}}+\frac{\lambda_{1} \lambda_{5}}{\mu_{1} \mu_{5}}+\frac{\lambda_{5} \lambda_{6}}{\mu_{5} \mu_{6}}+\frac{\lambda_{2} \lambda_{3} \lambda_{5}}{\mu_{2} \mu_{3} \mu_{5}}
\end{gathered}
$$

$$
\left.+\frac{\lambda_{1} \lambda_{3} \lambda_{5}}{\mu_{1} \mu_{3} \mu_{5}}+\frac{\lambda_{3} \lambda_{5} \lambda_{6}}{\mu_{3} \mu_{5} \mu_{6}}+\frac{\lambda_{3} \lambda_{4} \lambda_{5}}{\mu_{3} \mu_{4} \mu_{5}}\right)^{-1}
$$

A. Effect of Repair Rate of the Knotter $\left(\mu_{3}\right)$ and the Opener $\left(\mu_{5}\right)$

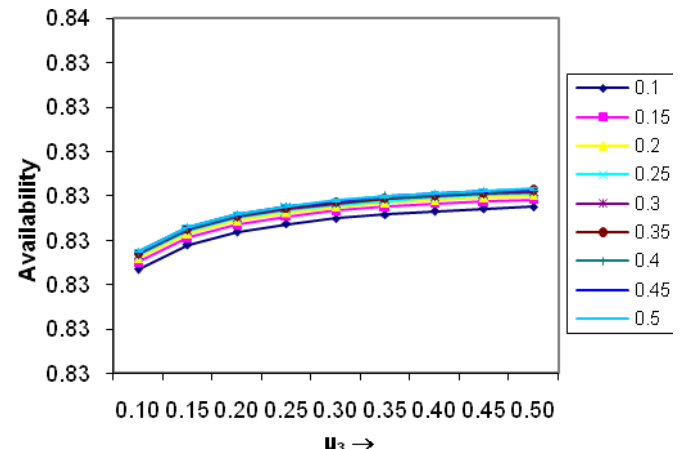

Fig. 3. The analysis of the table shows that repair rate of knotter insures more availability in comparison to repair rate of opener. 


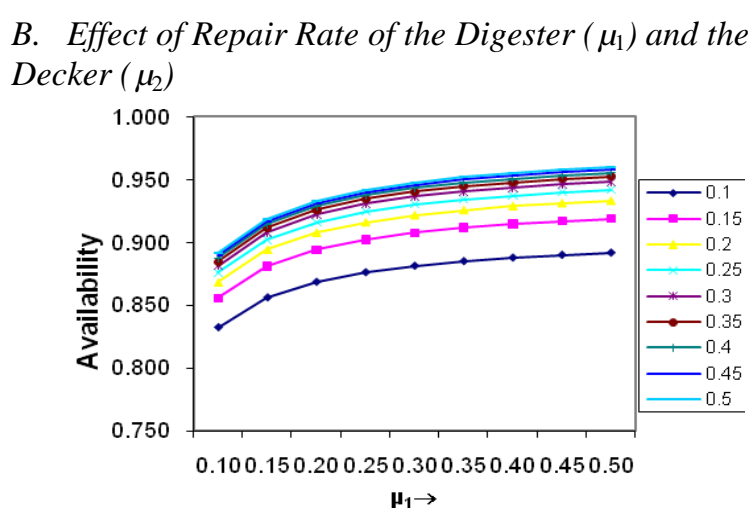

Fig. 4. The analysis of the table shows that repair rate of digester and decker insures equal availability.

\section{CONCLUSIONS AND FUTURE WORK}

The present paper can help in increasing the availability of the pulping system. The matrix method can be applied to complex systems that include a large number of differential equations. Using this system we can easily study the variation of availability with respect to time. Table I and Fig. 2 show the variation of availability with respect to time. Initially availability decreases sharply with respect to time and become almost stable after long duration of time.

The analysis of the system shows that the effect of repair rate of knotter has more impact on the availability of the system and also opener and the decker have little impact on the availability of the system. Thus care of knotter is more needed. Steady state behaviour of the system is discussed with the help of tables and graphs. Tables for repair rates are formed to study the behaviour of the system under different modes. The comparison of these availability tables help in location of weak systems and estimation of their contributions to the system availability. Various measures can be adopted to increase the availability of the system. Likewise we can study all the systems of the Paper industry. Since, all the systems are working in series in the Paper industry, the overall availability of the Paper industry can be evaluated from the following formula:

$$
\operatorname{Av}=\operatorname{Av}_{1}(t) \cdot \operatorname{Av}_{2}(t) \cdot \operatorname{Av}_{3}(t) \cdot \operatorname{Av}_{4}(t) \cdot \operatorname{Av}_{5}(t) \cdot \operatorname{Av}_{6}(t)
$$

where

$\operatorname{Av}_{1}(t)$ is the availability of the feeding system,

$\operatorname{Av}_{2}(t)$ is the availability of the pulping system,

$\operatorname{Av}_{3}(t)$ is the availability of the washing system,

$\operatorname{Av}_{4}(t)$ is the availability of the bleaching system,

$\operatorname{Av}_{5}(t)$ is the availability of the screening system and

$\operatorname{Av}_{6}(t)$ is the availability of the paper formation system.

The results are helpful for paper manufacturers and failures can be reduced through preventive maintenance, engineering modifications and careful attention to environmental conditions and operating loads.

\section{REFERENCES}

[1] B. S. Dhillon and C. Singh, Engineering Reliability - New Techniques and applications, John Willey and Sons, New York, 1981

[2] D. Kumar, I. P. Singh, and J. Singh, "Reliability analysis of the feeding system in the paper industry," Microelectron Reliability, vol. 28, no. 2, 1988, pp. 213-215.

[3] D. Kumar, J. Singh, and P. C. Pandey, "Availability analysis of the washing system in the paper industry," Microelectron Reliability, vol. 29, 1989, pp. 775-778.

[4] D. Kumar, J. Singh, and P. C. Pandey, "Availability of the crystallization system in the sugar industry under common-cause failure," IEEE Transactions on Reliability, vol. 41, no. 1, pp. 85-91, 1992.

[5] D. Kumar, J. Singh, and P. C. Pandey, "Operational behaviour and profit function for a bleaching and screening system in the paper industry," Microelectron Reliability, vol. 33, 1993, pp. 1101-1105.

[6] M. Zhao, "Availability for repairable components and series systems," IEEE Transactions on Reliability, vol. 43, no. 2, 1994.

[7] M. L. Shooman, "Reliability computation for systems with dependent failures," IEEE Annual Symposium on Reliability, pp. 1-7, 1996.

[8] Q. Michelson, "Use of reliability technology in the process Industry," Reliability Engineering and System Safety, pp. 179-181, 1998.

[9] J. Singh and P. Mahajan, "Reliability of utensils manufacturing plant - a case study," Opsearch, vol. 36, no. 3, pp. 260-269, 1999.

[10] H. F. Castro, Cavalco K, "Availability optimization with Genetic Algorithm," International Journal of Quality and Reliability Management, vol. 20, no. 7, 2003, pp. 847-863.

[11] P. C. Tewari, D. Joshi, and M. S. Rao, "Mathematical modeling and behavioural analysis of a refining system using genetic algorithm," in Proc. National Conference on Competitive Manufacturing Technology and Management for Global Marketing, Chennai, vol. 9, no. 1, 2005.

[12] G. Lal, R. K. Sharma, and J. Singh, "Behavioral study of the cement manufacturing plant-A numerical approach," Journal of Mathematics and Systems and Sciences, vol. 1, no. 1, pp. 50-69, 2005.

[13] G. Pawan, "Numerical analysis of reliability and availability of the serial process in butter-oil processing plant," International Journal of Quality and Reliability Management, pp. 303-306, 2005.

[14] J. Singh and Y. Goyal, "Availability and Behavior analysis of bread manufacturing plant," Journal of Mathematics and Systems Sciences, vol. 2, pp. 35-45, 2006.

[15] K. Ditlevson, "Availability, Reliability and downtime of system with repairable components," Reliability Engineering and System Safety, vol. 92, no. 2, pp. 66-72, 2007.

[16] R. Khanduja, P. C. Tewari, and D. Kumar, "Availability analysis of bleaching system of paper plant," Journal of Industrial Engineering, Udyog Pragati, vol. 32, pp. 24-29, 2008.

[17] G. Sanjeev, Y. Goyal, and J. Singh, "Analysis of agriculture based industry - A short cut approach," JMASS, vol. 5, no. 2, pp. 95-109, 2009.

[18] E. Zio and J. K. Vaurio "Maintenance modeling and applications," Reliability Engineering and System Safety, vol. 94, no. 1, pp. 1.

Zeenat Zaidi is working as an assistant professor (Statistics) in Al- Qassim University, Kingdom of Saudi Arabia (K.S.A). She has total experience of more than seven years of teaching applied mathematics. She has completed her $\mathrm{Ph} . \mathrm{D}$. on the topic "Mathematical Techniques for cost and profit analysis of some process industries" in 2012. She has written ten research papers related to different process industries. Among them seven are published in reputed journals and three are communicating. She has presented three articles and five papers in National seminars and International conferences. Her primary research interest is performance Improvement, the mathematical modeling and analysis of process industries through Reliability Engineering.

Yogesh Kumar Goyal is presently working as an associate professor at Aggarwal P.G. College Ballabgarh, Haryana. He has published seven books and twelve research papers. He has a rich experience in teaching and academics of more than 35 years. Dr. Goyal has guided one M.Phil. and one $\mathrm{Ph}$.D. scholars in the field of Algebra and Reliability and one research scholar are presently working with him. 